\title{
Fat suppression techniques in breast magnetic resonance imaging: a critical comparison and state of the art
}

This article was published in the following Dove Press journal:

Reports in Medical Imaging

16 March 2015

Number of times this article has been viewed

\section{Chen Lin \\ Clark David Rogers \\ Shadie Majidi}

Department of Radiology and Imaging Science, Indiana University School of

Medicine, Indianapolis, IN, USA
Correspondence: Chen Lin Department of Radiology and Imaging Science, Indiana University School of Medicine, 950 West Walnut St R2 EI24, Indianapolis, IN 46202, USA

Tel +l 3 I 72786572

Email clin I@iupui.edu
Abstract: Robust and accurate fat suppression is highly desirable in breast magnetic resonance imaging (MRI) because it can considerably improve the image quality and lesion conspicuity. However, fat suppression is also more challenging in the breast compared with other regions in the body. Technical advances have been made over time to make fat suppression more efficient and reliable. Combined with other innovations, breast MRI continues to be the most sensitive and comprehensive diagnostic modality in the detection and evaluation of breast lesions. This review offers a critical comparison of various fat suppression techniques in breast MRI including spectral-selective excitation and saturation techniques based on the chemical shift difference between fat and water, the inversion recovery techniques based on the T1 relaxation time difference, the hybrid spectral-selective inversion recovery techniques, and the new Dixon fat and water separation techniques based on the phase difference between fat and water signal at different echo times. This review will also cover less frequently used techniques such as slice-selective gradient reversal. For each fat suppression technique in breast MRI, a detailed explanation of the technical principle, the advantages and disadvantages, the approaches for optimization as well as the clinical examples are included. The additional challenges of fat suppression in breast MRI at higher field strength and in the presence of metallic and silicone implants are also discussed.

Keywords: breast MRI, fat suppression, dynamic contrast enhanced imaging, diffusion weighted imaging, magnetic resonance spectroscopy

\section{Introduction}

\section{The importance of breast MRI}

Breast cancer is the second most common cancer in women in the US. It is also a leading cause of mortality among women. ${ }^{1} \mathrm{~A}$ woman living in the US have a $12.3 \%$ (1 in 8 ) lifetime risk of being diagnosed with breast cancer. Early detection as well as improvements in treatment have resulted in a reduction in breast cancer mortality. Therefore, it is very important for women to follow recommended screening guidelines in order to detect breast cancer at an earlier stage of the disease. ${ }^{2}$

Breast magnetic resonance imaging (MRI) is highly sensitive in detection and characterization of primary and recurrent breast cancer. ${ }^{3}$ In patients with known malignancies, breast MRI improves the assessment of the extent of disease, helps in preoperative staging and subsequent choice of therapy, and has been found to be beneficial in the assessment of response to neoadjuvant chemotherapy. ${ }^{4-6}$ Several studies have demonstrated higher sensitivity for breast MRI screening compared with mammography and breast ultrasound in high-risk women. There is evidence that 
screening of the contralateral breast with MRI will detect occult malignancy in approximately $3 \%$ to $9 \%$ in women with newly diagnosed breast cancer. ${ }^{7}$

\section{Overview of breast MRI}

A typical breast MRI exam includes a non-fat-suppressed T1-weighted scan, a fat-suppressed T2-weighted (T2W) scan and a dynamic contrast enhanced (DCE) scan. Recent studies have also demonstrated the value of diffusion weight imaging (DWI) and magnetic resonance spectroscopy (MRS) in breast MRI. Although DWI and MRS offer additional information which could be helpful in the diagnosis and differentiation of certain breast lesions, they are not routinely used in clinical breast MRI due to their limitations and technical challenges.

\section{$\mathrm{T} 2 \mathrm{~W}$ of breast}

T2W imaging is an essential MRI technique that can provide much information to help distinguish benign from malignant lesions. Lesions with greater water content generally appear bright on T2W images and are more likely to be benign than malignant. For example: benign lesions such as cysts appear bright on T2W images. While adenocarcinoma does not appear bright on $\mathrm{T} 2 \mathrm{~W}$, some mucinous and necrotic breast tumors can appear bright. A lesion with rim enhancement can be confirmed to be a cyst if it is bright on T2W; similarly, fibroadenomas and lymph nodes which may mimic carcinoma in contrast enhancement kinetics can be determined to be benign based on their bright signal on $\mathrm{T} 2 \mathrm{~W}$.

\section{DCE-MRI of breast}

In breast MRI exam, dynamic contrast enhanced scan (DCEMRI) is the most critical component of the test. ${ }^{8,9}$ DCE-MRI provides both morphological and functional (ie, perfusion) information, both critical for differentiating between benign and malignant lesions ${ }^{10}$ it is therefore very helpful in characterizing primary and recurrent breast cancers as an adjunct to mammography and ultrasonography. Breast MRI in highrisk women has been shown to have a higher sensitivity than mammography, and the combination of mammography and MRI in this population has the highest sensitivity. Overall, studies on high-risk populations have found the sensitivity for DCE-MRI ranging from $71 \%$ to $100 \%$ versus $16 \%$ to $40 \%$ for mammography. ${ }^{11}$ Because of its high sensitivity to detect breast abnormalities, DCE-MRI is the leading MRI technique for both the initial diagnosis and the treatment monitoring of malignant lesions.

\section{DWI of breast}

In an effort to improve the specificity of breast MRI, more advanced techniques such as DWI are being considered. Currently, DWI is not routinely used for breast MRI but studies suggest that it can be helpful in differentiating benign from malignant lesions. ${ }^{12,13}$ DWI is based on differences in the motion of water molecules in tissue that is the self-diffusion of water protons. High cellular density in malignant tumors due to rapid proliferation leads to restricted ability of the water to diffuse freely within the tissue. Using DWI, one is able to calculate the apparent diffusion coefficient, which is a quantitative measure directly proportional to the diffusion of water. Thus, malignant tumors will have a reduced apparent diffusion coefficient due to their restricted diffusion and can be distinguished from less cellular, benign tumors.

\section{MRS of breast}

MRS is another advanced MRI technique that will continue to improve with the advances in stronger field magnets. The basis of MRS is the use of the metabolic profile of tissue to make diagnostic determinations. Specifically, it has been observed that the level of choline and its metabolites is elevated in tumors compared to benign tissue. ${ }^{14}$ Unfortunately, there are confounding factors such as the fact that lactating women may also show elevated choline. ${ }^{15}$ More research is needed for a better understanding of MRS application for breast MRI.

\section{The need for fat suppression in breast MRI}

Fat suppression is an essential and integral part of a breast MRI exam. While a set of non-fat-suppressed T1-weighted images is typically included in breast MRI to visualize the anatomy and the distribution of fatty tissue, all the key breast MRI scans, including T2W, DCE, DWI, and MRS, require or benefit from fat suppression.

Fat appears bright in T2W MRI images because of its long $\mathrm{T} 2$ relaxation time. The fat signal, if not suppressed, could mask the features of interest and interfere with the evaluation of benign lesions. As shown in Figure 1, the conspicuity of fibroglandular tissue and lymph node is greatly improved in the breast with complete fat suppression compared to the contralateral breast where fat suppression is non-uniform. Unsuppressed fat signal can also cause chemical shift artifact, which appears as a thin band of high or low signal at fat and soft tissue boundaries.

Fat also appears bright in DCE-MRI due to its relatively short $\mathrm{T} 1$ relaxation time. It is likely to interfere 


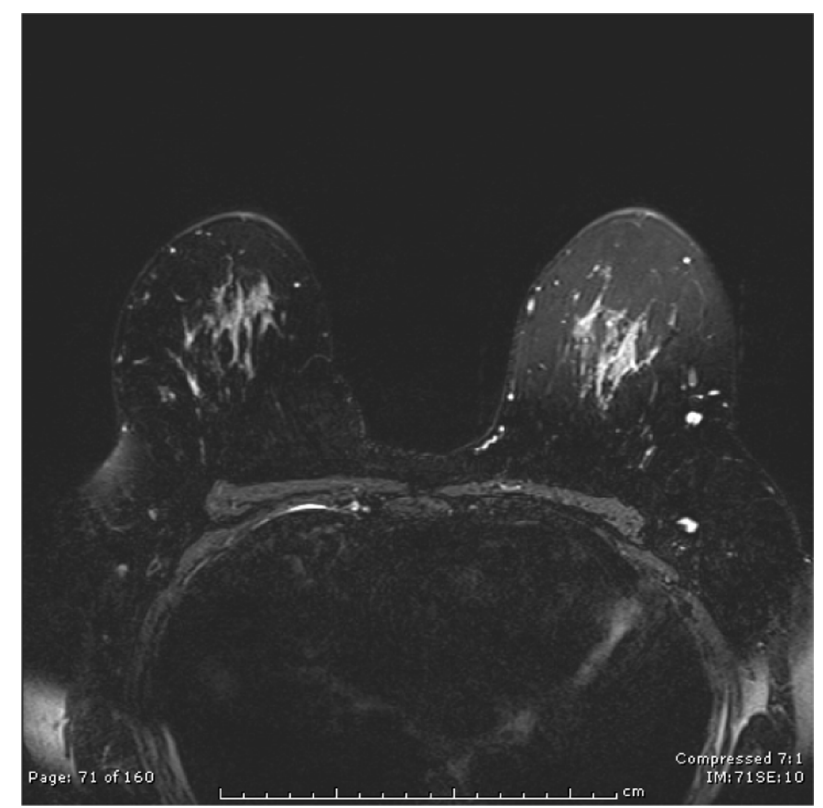

Figure I Bilateral axial T2-weighed breast magnetic resonance image.

Notes: The left breast demonstrates incomplete fat saturation. The right breast shows the improved contrast between background fat and the glandular tissue and lymph nodes resulting from complete fat suppression.

with the evaluation of tissue signal changes from dynamic contrast enhancement or obscure abnormal areas of contrast enhancement. Therefore, it is very important to suppress fat tissue signal to improve the detection of enhancing lesions. As shown in Figure 2, the image quality was improved when fat suppression works correctly.

Because fatty tissue does not take up contrast, its signal should remain constant over the course of contrast uptake and washout. Therefore, in the ideal situation, fat signal in the post-contrast images can be cancelled out by performing subtraction with the corresponding pre-contrast images.
However, this is rarely the case in clinical patient exams. A certain degree of patient motion is unavoidable over the course of 6-10 minutes breast DCE-MRI acquisition since a patient has to lie down in an uncomfortable prone position during the exam. The change in breast shape and position over time will also cause mis-registration and introduce errors in the subtraction. The un-subtracted fat signal can produce patterns which actually mimic contrast enhancement. ${ }^{16}$ Therefore, fat suppression is usually necessary in DCE-MRI.

Another reason for fat suppression in breast DCE-MRI is to reduce the error in pharmacokinetic modeling in order to quantify kinetic parameters such as the transfer constant and the fractional volume of extravascular extracellular space. For example, in the commonly used two-compartment model, the fat signal is not accounted for because the tissue is mottled with the vascular and extracellular compartments. Even though fat signal is a relatively small fraction compared with the signal from the contrast-enhanced glandular tissue, it can still skew the results in quantitative pharmacokinetic modeling. ${ }^{17}$

Fat suppression is also critical in breast DWI, which is based on an echo planar imaging (EPI) sequence for data acquisition. Being a single-shot gradient echo sequence, chemical shift can cause a large displacement of fat signal in the phase-encoding direction of EPI. Therefore, fat suppression is always used in DWI throughout different regions in the body. Fat suppression is even more important for breast DWI since there is a large amount of fat in the breast.

In breast MRS, the resonance of total choline (tCho) at $3.2 \mathrm{ppm}$, which includes the contributions from
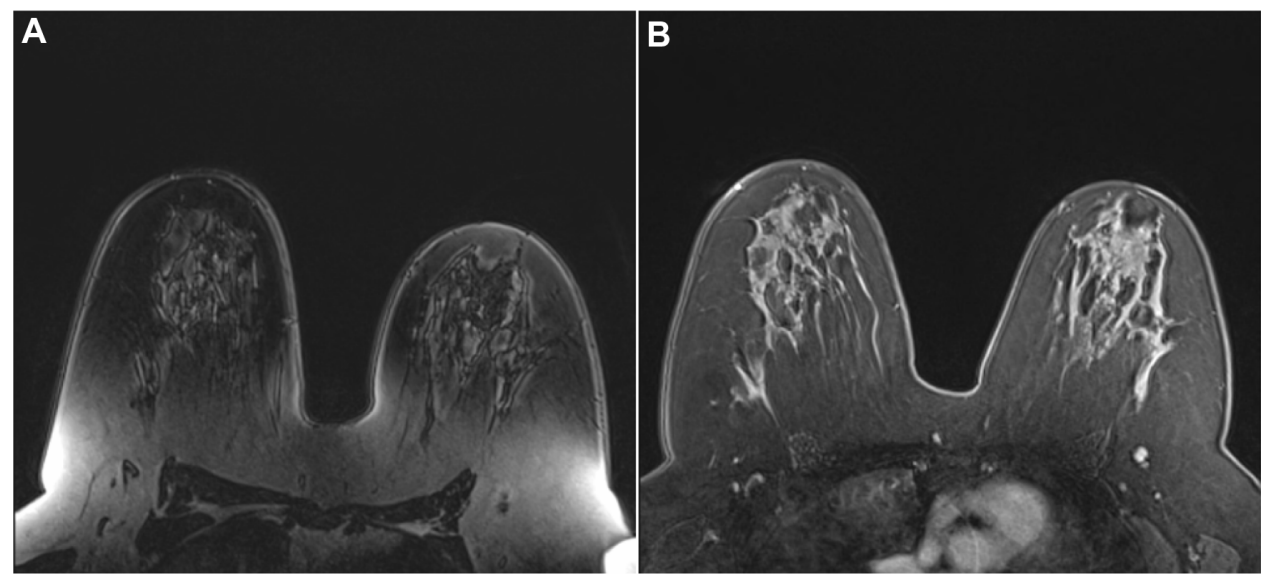

Figure $2 \mathrm{TI}$-weighted images of the left and right breast.

Notes: TI-weighted image with inhomogeneous fat saturation $(\mathbf{A})$ which was subsequently repeated to improve fat suppression. The repeated image (B) shows improved visibility of anatomical details. 
multiple choline containing metabolites such as choline, phosphocholine, and glycerophosphocholine has been associated with malignancy. If a breast MRS voxel contains a large amount of fat, the sideband of lipid resonance at $1.3 \mathrm{ppm}$ could mask the tCho peak. Fat suppression is usually applied in breast MRS to attenuate the lipid signal and improve the sensitivity and accuracy of tCho detection. It is based on the same reason that water suppression is often applied in MRS to suppress the dominating signal from water protons in order to detect metabolites of much lower concentration.

\section{The challenges of fat suppression in breast MRI}

While fat suppression is necessary or highly desirable in different breast MRI scans, there are unique challenges for fat suppression in breast MRI. First of all, because the breast has large amounts of fat, fat suppression technique for breast MRI must be highly effective in order to completely remove fat signal. Second, the breasts are surrounded by air, and the magnetic susceptibility difference between the breast tissue and air causes local magnetic field $\left(\mathrm{B}_{0}\right)$ inhomogeneity. Therefore, fat suppression technique for breast MRI should also have high tolerance for $\mathrm{B}_{0}$ inhomogeneity. Third, high temporal resolution is needed in breast DCE-MRI in order to properly sample the time curve of contrast enhancement, ie, the contrast wash-in and wash-out. To achieve high temporal resolution, the fat suppression cannot take up too much time and slow down the acquisition. Finally, when imaging patients with silicone implants, it is important to suppress only the fat signal without affecting the silicone signal, so that the presence and distribution of silicone can be visualized. This is difficult, because the resonance frequencies of fat and silicone are very close to each other.

\section{The desired quality of fat suppression}

Despite these challenges, fat suppression in breast MRI should be accurate and robust. A failure in fat suppression could negatively impact the image quality and distort the contrast uptake curve. ${ }^{18}$ Accurate fat suppression means the fat signal is completely removed while the water signal is preserved. Robust fat suppression means fat suppression should be highly accurate throughout the entire breast and surrounding regions of interest, such as the axilla, and be consistent on subsequent imaging exams. To meet these challenges and improve the quality of breast MRI, new and more advanced fat suppression techniques have been developed and refined over time, allowing us to gradually approach these goals. Although fat suppression in breast MRI is an important issue and an active area of research and development, there has been no comprehensive review dedicated to this topic.

\section{Methods}

\section{Fat suppression methods in breast MRI}

MRI signal of fat has its unique characteristics. Its resonance frequency is different from that of water signal due to chemical shift. The frequency separation between fat and water signal is about $220 \mathrm{~Hz}$ at $1.5 \mathrm{~T}$ and $440 \mathrm{~Hz}$ at $3.0 \mathrm{~T}$ as illustrated schematically in Figure 3. Fat signal also has a relative short $\mathrm{T} 1$ relaxation time $(-300 \mathrm{~ms}$ at $1.5 \mathrm{~T}$ and $\sim 370 \mathrm{~ms}$ at $3.0 \mathrm{~T})$ and long $\mathrm{T} 2$ relaxation time $(\sim 50 \mathrm{~ms})$ which makes fat hyper-intense on both T1-weighted and T2W images. ${ }^{19}$ Based on these properties, different techniques have been developed to selectively attenuate fat signal in breast MRI. A brief summary of pros and cons of commonly used fat suppression techniques is provided in Table 1.

\section{Chemical shift selective saturation or Quick FatSat}

A commonly used method for fat suppression is based on the difference in resonance frequency between fat and water signal (ie, the chemical shift difference). As illustrated in Figure 4, after applying a spectral-selective radiofrequency (RF) pulse with a frequency offset matching the resonance frequency of fat protons $(220 \mathrm{~Hz}$ at $1.5 \mathrm{~T})$, only the fat magnetization will be excited and tipped into the transverse plane. Such transverse magnetization is then de-phased by a crushing gradient pulse immediately afterward so that it would not produce any signal. On the other hand, the water magnetization is not affected by the spectral-selective RF pulse.

In order for the chemical shift selective saturation (CHESS) fat suppression ${ }^{20}$ to work effectively, the resonance frequency of fat must be within the narrow bandwidth of the spectralselective RF pulse across the entire field of view (FOV). In case the main $\mathrm{B}_{0}$ is not uniform, the fat resonance frequency, which

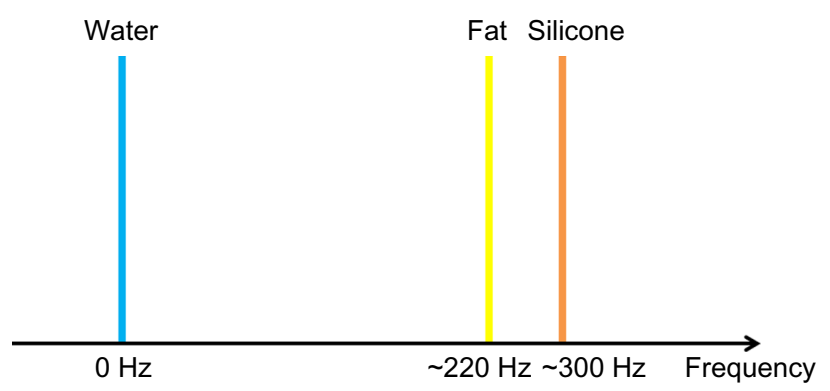

Figure 3 A schematic spectrum at I.5T of water, fat, and silicone.

Notes: Fat and water peaks are separated by about $220 \mathrm{~Hz}$. Fat and silicone peaks are separated by about $80 \mathrm{~Hz}$. These differences increase linearly with magnetic field $\left(B_{0}\right)$. 
Table I A high-level summary of the relative advantages and disadvantages of fat suppression techniques in breast magnetic resonance imaging

\begin{tabular}{llll}
\hline $\begin{array}{l}\text { Fat } \\
\text { suppression } \\
\text { technique }\end{array}$ & $\begin{array}{l}\text { Sensitivity to } B_{0} \\
\text { inhomogeneity }\end{array}$ & $\begin{array}{l}\text { Sensitivity to } B_{1}^{+} \\
\text {inhomogeneity }\end{array}$ & $\begin{array}{l}\text { Impact } \\
\text { to SNR } \\
\text { efficiency }\end{array}$ \\
\hline CHESS & -- & - & - \\
STIR & ++ & - & - \\
SPIR/SPECIAL/ & - & + & - \\
SPAIR & & & + \\
DIXON/IDEAL & ++ & ++ & +
\end{tabular}

Notes: "+" implies slight advantage and "++" implies strong advantage, while "-" implies slight disadvantage and "--_" implies strong disadvantage.

Abbreviations: CHESS, chemical shift selective saturation; STIR, short tau inversion recovery; SPIR, spectral presaturation with inversion recovery; SPECIAL, spectral inversion at lipid; SPAIR, spectrally-selective adiabatic inversion recovery; $\mathrm{B}_{0}$, magnetic field; IDEAL, Iterative Decomposition of water and fat with Echo Asymmetry and Least-squares estimation; SNR, signal to noise ratio.

is proportional to $\mathrm{B}_{0}$, will also vary. Fat suppression would fail if the fat frequency is outside of the bandwidth of the saturation RF pulse. In a worse scenario, the water frequency may be shifted into the saturation band of the RF pulse at certain locations, causing the water signal to be suppressed instead of fat as shown by the example in Figure 5.

The CHESS fat suppression module, which consists of a saturation RF pulse and a crushing gradient pulse, does add a significant amount of additional time compared to the gradient echo acquisition itself. It would prolong the scan time or reduce the temporal resolution of dynamic acquisition. One way to avoid such problem is to apply the fat suppression module only prior to the acquisition of central k-space data points. Since the image contrast is predominately determined by the central k-space data, interleaved fat suppression (QFS) can dramatically reduce the scan time while maintaining the benefit of fat suppression. ${ }^{21}$ However, QFS module in fast gradient echo acquisition also alters the regular repetition of excitations and disrupts the steady state of magnetization. As a consequence, the signal intensity does not follow the well-established simple model for steady state, making the qualitative analysis more challenging. ${ }^{21}$

\section{Short tau inversion recovery}

Another approach for fat suppression is based on the $\mathrm{T} 1$ relaxation time difference between fat and water protons. By acquiring signal at a specific delay time $(150 \mathrm{~ms}$ for $1.5 \mathrm{~T}$ and $200 \mathrm{~ms}$ for 3.0T) after a non-spectral-selective (broadband) inversion RF pulse, the fat signal is nulled as illustrated in Figure 6. Since short tau inversion recovery (STIR) fat suppression does not rely on spectral-selective RF pulse, it is immune to $\mathrm{B}_{0}$ inhomogeneity.

There are several drawbacks with the STIR technique. First of all, at the null point for fat, water magnetization is only partially recovered. Therefore, the water signal is also attenuated ${ }^{22}$ and becomes T1-weighted after inversion recovery (IR). Another drawback with STIR fat suppression is that the IR process takes an even longer time than the CHESS module. Because it is time consuming, STIR fat suppression is only used in static acquisitions such as T2W imaging and sometimes diffusion-weighted imaging of the breast. Finally, although STIR is insensitive to $\mathrm{B}_{0}$ variations, it can be affected by variation of $\mathrm{RF}$ amplitude $\left(\mathrm{B}_{1}^{+}\right)$. If the inversion RF pulse does not uniformly produce an exact 180 degree flip angle across the FOV, the delay time (time of inversion [TI]) to null fat signal would also be different from
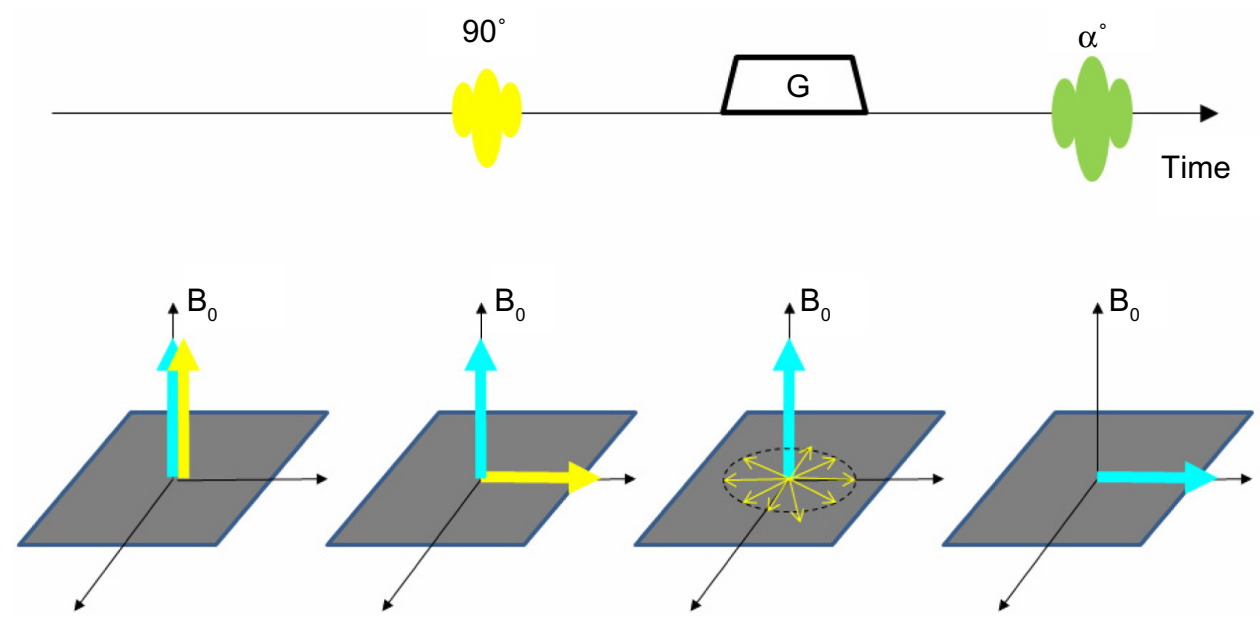

Figure 4 CHESS fat suppression.

Notes: In CHESS fat suppression, a spectral-selective pulse tuned to the fat resonance tips the fat magnetization (represented by yellow arrows) to transverse plane (frame 2). The fat magnetization is then dephased by a gradient pulse $(\mathrm{G})$ so the net magnetization is zero and only the water magnetization (represented by blue arrows) is remaining (frame 3). In the following excitation, only water magnetization is tipped to the transverse plane to eventually produce MR signal.

Abbreviations: CHESS, chemical shift selective saturation; $M R$, magnetic resonance; $B_{0}$, magnetic field. 


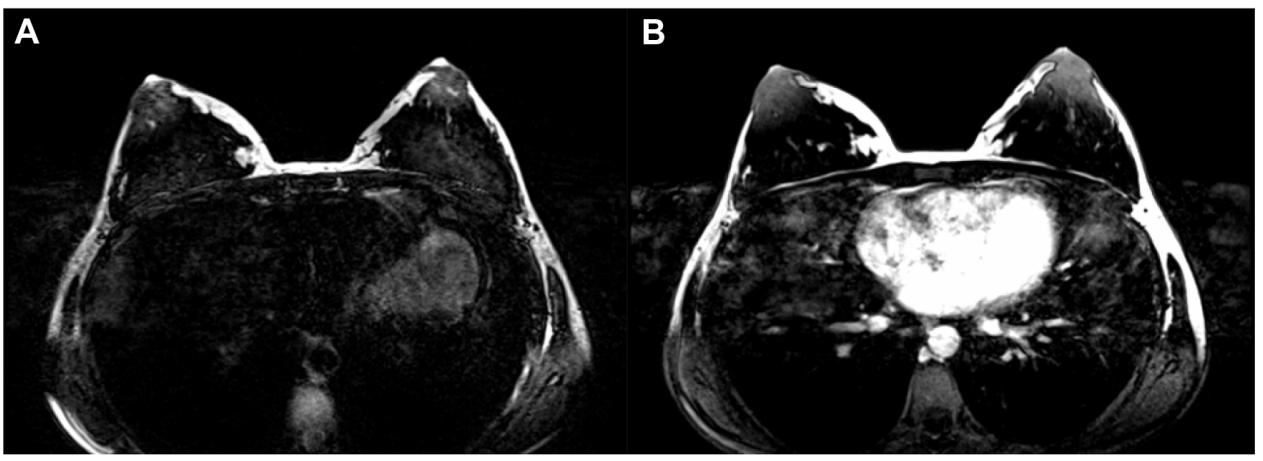

Figure 5 Incorrect setting of RF pulse frequency in spectral-selective fat suppression techniques such as CHESS can cause serious problems in breast MRI. Notes: In this example, it appears that the frequency of fat suppression RF pulse was set to water resonance frequency instead of fat, therefore suppressing the signal from glandular tissue instead of fat in both T2W (A) and DCE (B) images.

Abbreviations: CHESS, chemical shift selective saturation; MRI, magnetic resonance imaging; T2W, T2-weighted; DCE, dynamic contrast enhanced; RF, radio frequency.

the nominal value and certain regions may then have incomplete fat suppression. Because dielectric effect is more prominent at higher field strength, $\mathrm{B}_{1}^{+}$inhomogeneity is also worse at $3 \mathrm{~T}$ and $7 \mathrm{~T}$ compared to $1.5 \mathrm{~T}$. The additional challenges for fat suppression at higher field strengths will be discussed in detail in Fat suppression at $3.0 \mathrm{~T}$ versus $1.5 \mathrm{~T}$.

\section{Spectral presaturation with inversion recovery, spectral inversion at lipid, and spectrally-selective adiabatic inversion recovery}

Inversion recovery based fat suppression technique (STIR) also attenuates water signal and alters image contrast if a non-selective inversion pulse is used. Such a problem can be avoided by making the inversion spectrally selective to fat so that only the fat magnetization would be inverted and subsequently nulled after a certain delay time. Since the water magnetization is not affected by the inversion pulse tuned to fat resonance, the signal to noise ratio and image contrast are preserved, as illustrated in Figure 7. Such fat suppression method is known as SPIR and SPECIAL. To reduce the time of IR process, a less than $180^{\circ}$ flip angle, typically $100^{\circ}-120^{\circ}$, and a corresponding shorter TI are often used in SPIR and SPECIAL.

Another problem with IR-based fat suppression technique is that it may suffer from $\mathrm{B}_{1}^{+}$inhomogeneity, as discussed previously. Spectrally-selective adiabatic inversion recovery (SPAIR $)^{23}$ was developed to address this issue. By substituting the conventional RF pulse with an adiabatic RF pulse, the inversion is made more robust and not sensitive to $\mathrm{B}_{1}^{+}$ variation, as illustrated in Figure 7. As a result, SPAIR typically produces more uniform fat suppression than CHESS and STIR, especially at higher field strengths such as $3 \mathrm{~T}$, as
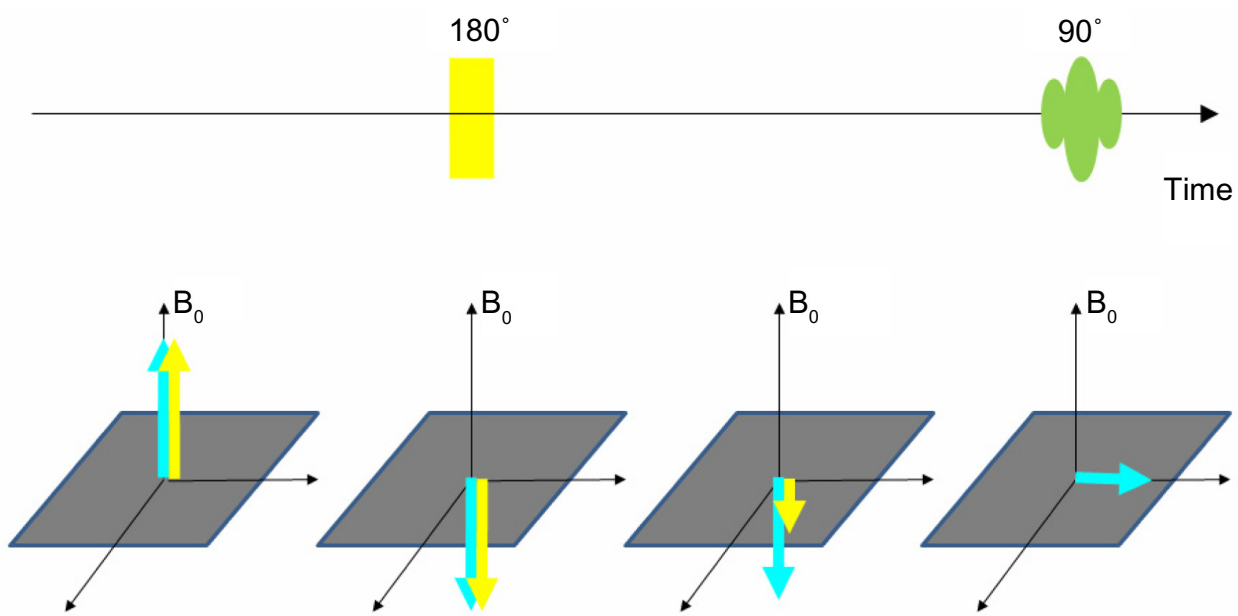

Figure 6 STIR fat suppression.

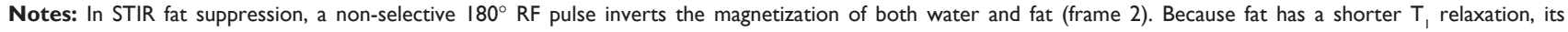
magnetization recovers faster than water (frame 3). After a certain time, the fat magnetization reaches zero (ie, nulled). If the excitation pulse is played out at this moment; only the remaining water magnetization will produce a signal.

Abbreviations: STIR, short tau inversion recovery; $\mathrm{B}_{0}$, magnetic field; RF, radio frequency. 


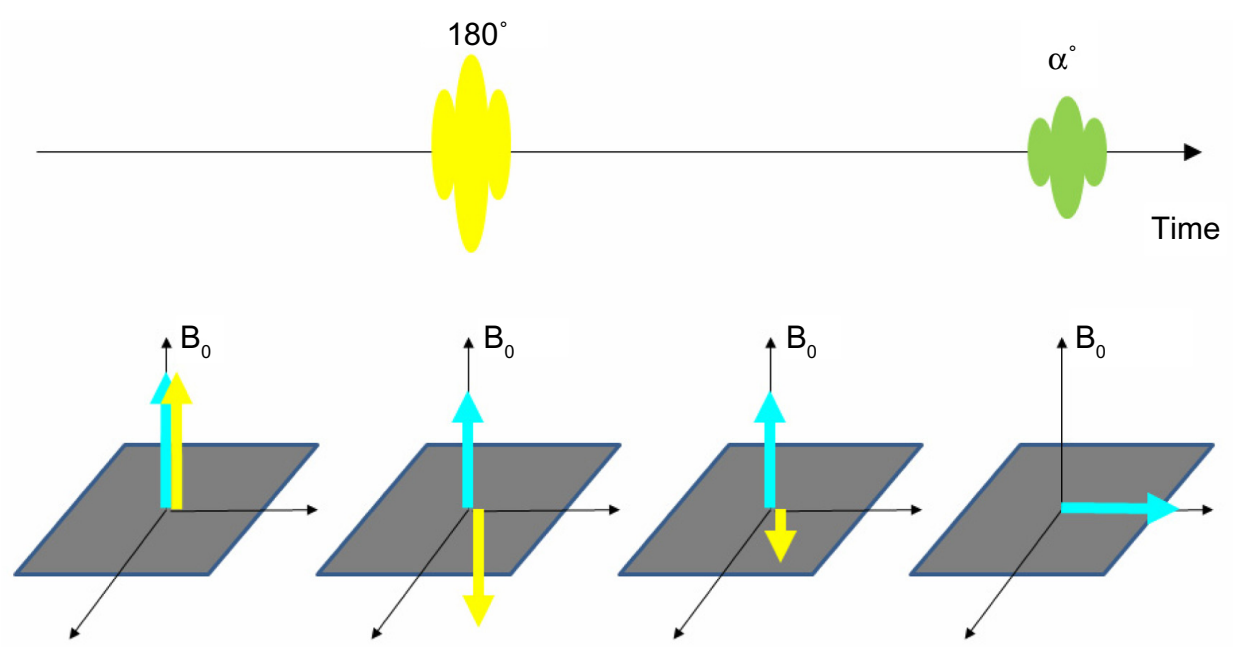

Figure 7 Replacing non-spectral-selective inversion RF pulse in STIR with an adiabatic and spectral-selective RF pulse matching fat resonance frequency.

Notes: By replacing the non-spectral-selective inversion RF pulse in STIR with an adiabatic and spectral-selective RF pulse matching fat resonance frequency, the inversion only affects fat magnetization (frame 2). While fat magnetization goes through the inversion recovery process and is nulled after a certain delay time, the water magnetization remains unchanged. With an adiabatic pulse, the inversion is more robust with respect to $\mathrm{B}_{1}^{+}$variation.

Abbreviations: STIR, short tau inversion recovery; $B_{0}$, magnetic field; RF, radio frequency.

shown in Figure 8. To overcome the long duration associated with IR, SPIR, SPECIAL, or SPAIR, fat suppression modules can also be applied in an interleaved fashion, only prior to the acquisition of central k-space data in a dynamic acquisition.

\section{Spectral-selective (water) excitation with composite pulse and spectral spatial pulse}

Spectral-selective excitation can be achieved using a composite RF pulse which consists of several sub-pulses with specific inter-pulse delay. As illustrated in Figure 9, binomial pulse is one example of such composite excitation pulse. By selectively exciting water resonance, ie, water excitation (WE), the fat signal is suppressed. The spectral selectivity or the sharpness of the excitation profile in the spectral domain improves with increas- ing number of sub-pulses, but the length of the composite pulse also increases. Therefore, $(1,1)$ binomial pulse (Water Excitation, Siemens, Erlangen Germany) and $(1,2,1)$ binominal pulse (ProSet, Philips, Best, Netherlands) are typically used in clinical applications. Advanced binomial excitation pulse design with no inter-pulse delay has also been developed for fast fat suppression in dynamic breast imaging and has been shown to provide WE with excellent fat suppression in minimum time. ${ }^{24}$

Spectral spatial (SPSP) pulse is another type of composite pulse which achieves spectral-selective excitation through the modulation of the RF amplitude and phase in each sliceselective sub-pulse. ${ }^{25,26}$ An example of SPSP pulse and its excitation profile are shown in Figure 10. SPSP pulse typically has more than ten sub-pulses and has relatively long pulse duration. Its application is mostly limited to diffusion
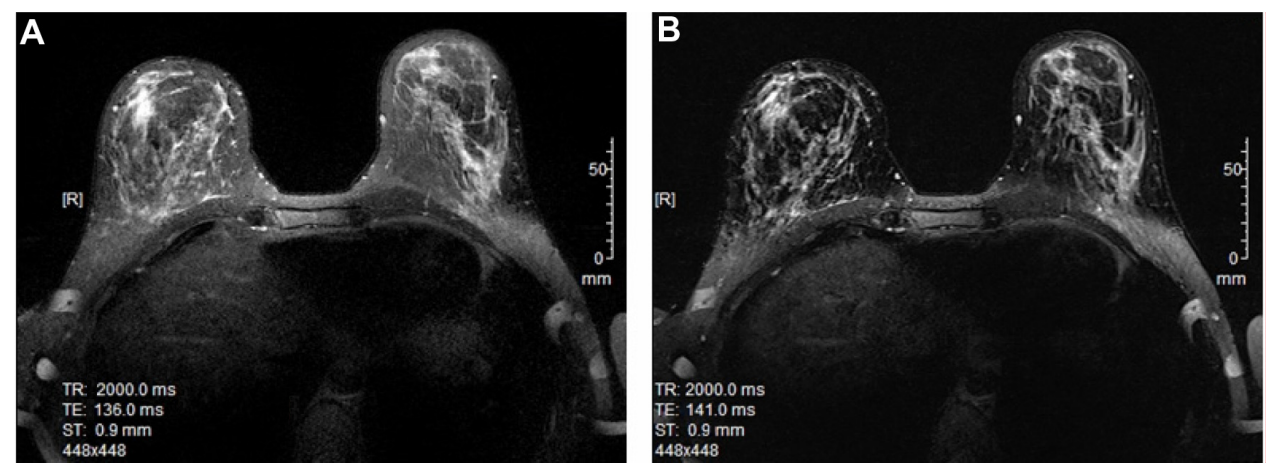

Figure 8 Three-dimensional T2-weighted breast images.

Notes: Three-dimensional T2-weighted breast images at 3.0T with interleaved CHESS fat suppression (QFS) (A) and with interleaved SPAIR fat suppression (B). Better image contrast with more complete fat suppression was achieved with SPAIR fat suppression. $448 \times 448$ is the matrix size of both images.

Abbreviations: CHESS, chemical shift selective saturation; QFS, Quick FatSat; SPAIR, spectrally-selective adiabatic inversion recovery; TE, echo time; TR, repetition time; ST, slice thickness. 

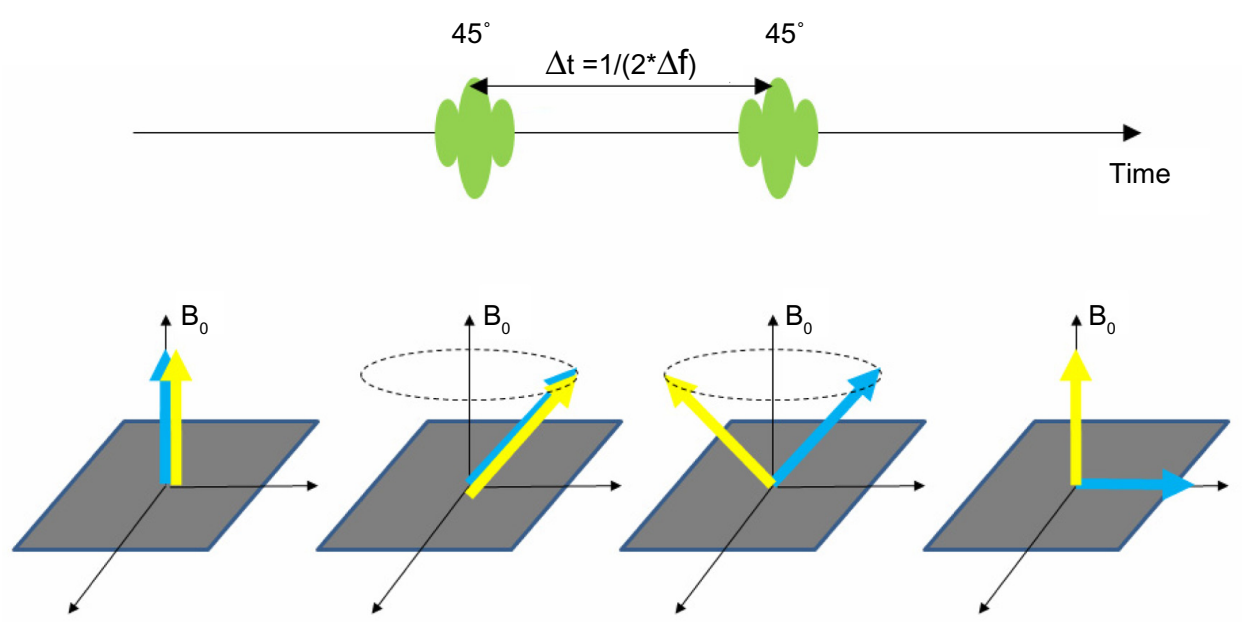

Figure 9 An example of (I, I) binomial pulse.

Notes: Immediately after a $45^{\circ} \mathrm{RF}$ pulse is applied, the magnetizations of fat and water are in-phase (frame 2 ). After a delay ( $\Delta \mathrm{t}$ ) equaling the inverse of two times the frequency difference between fat and water $(\Delta f)$ the water and fat magnetizations become out-of-phase (frame 3 ), then if another $45^{\circ} \mathrm{RF}$ pulse is applied, the water magnetization would effectively experience a $90^{\circ} \mathrm{RF}$ pulse as a typical excitation. On the other hand, the fat magnetization would return to original state.

Abbreviations: $B_{0}$, magnetic field; RF, radio frequency.

weight spin-echo (EP)-EPI where such long excitation pulse duration does not have a negative impact. Recently, dualband spectral-spatial excitation, which excites two parallel slabs, has been demonstrated to provide homogeneous fat suppression in bilateral breast imaging when combined with independent shims. ${ }^{27}$

\section{Dixon/Iterative Decomposition of water and fat with Echo Asymmetry and Least- squares estimation (IDEAL) fat and water separation}

Dixon Fat and Water Separation Technique, ${ }^{28,29}$ as illustrated in Figure 11, relies on the fact that there is an increasing phase difference between fat and water signal after the initial excitation because of their chemical shift difference. Such phase difference would cause the fat and water signals to be in-phase or out-of-phase with respect to each other depending on the echo time (TE) in a gradient echo sequence. By acquiring mixed fat and water signals at different TEs, one can separate the fat and water signals to generate water-only and fat-only images.

While acquiring data at two different TEs (two-point Dixon) is adequate for separating fat and water signals, additional echoes from more TEs can improve the stability of fat and water separation and also increase the SNR at the expense of longer acquisition time. For example, three-point DIXON
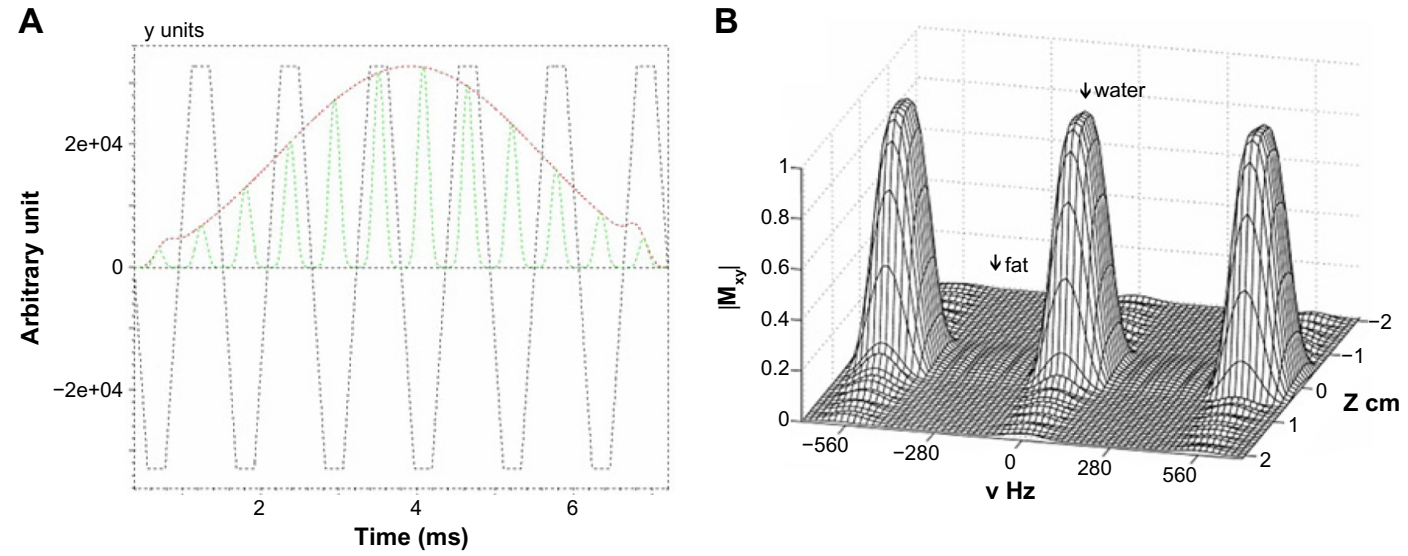

Figure 10 The typical composition of a spectral spatial pulse and the simulation results demonstrating both spectral and spatial selective excitation.

Notes: An SPSP pulse consists of a series of slice-selective excitation pulses with the amplitude modulated (A). Such a composite pulse produces an excitation profile which is not only slice-selective, but also has periodic excitation and bands in the spectral domain (B). By designing an SPSP pulse with water resonance in the middle of an excitation band and fat resonance between two excitation bands, only the water magnetization would be excited.

Abbreviation: SPSP, SPectral SPatial pulse. 

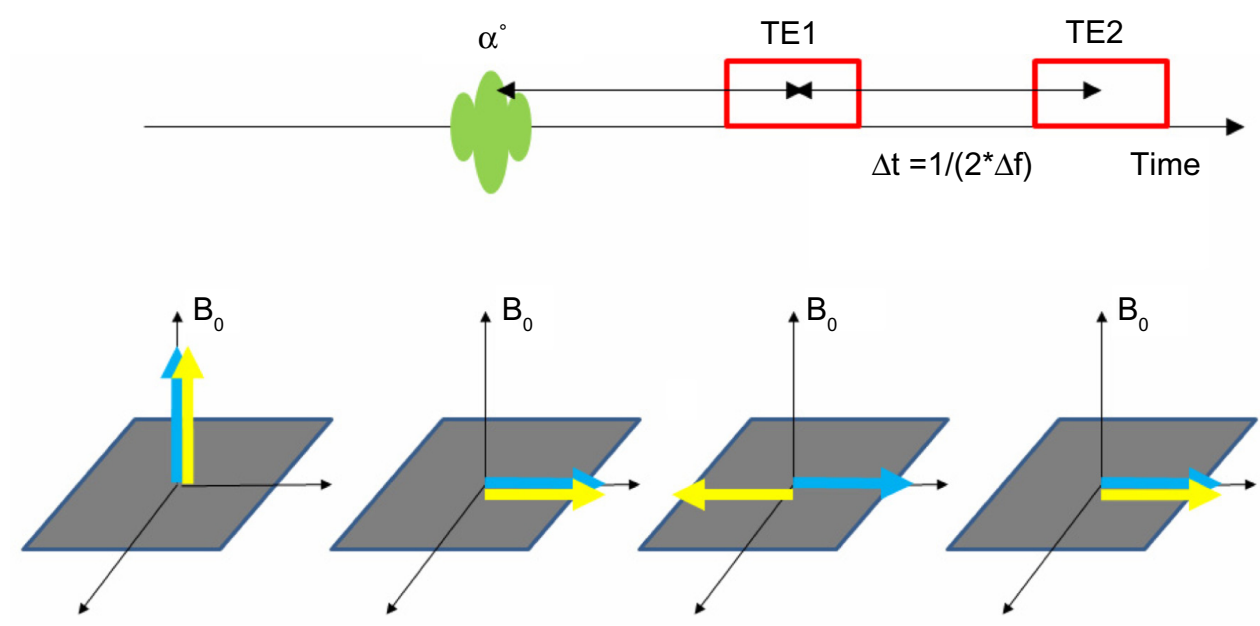

Figure II Two-point Dixon Technique

Notes: After the initial excitation (frame 2), two gradient echoes at different echo times (TEs) are acquired in two-point Dixon, the magnetizations of fat and water would be opposed-phase in one echo (ie, frame 3) and in-phase in another echo (ie, frame 4). The water-only and fat-only images can be generated from the combination of in-phase and opposed-phase data sets. A new Dixon fat and water separation algorithm has been developed which relaxes strict in-phase and opposed-phase requirements.

Abbreviation: $B_{0}$, magnetic field; $\Delta t$, delay between echoes; $\Delta f$, frequency difference between fat and water.

technique has been shown to provide better fat suppression and signal homogeneity than SPAIR and ProSet. ${ }^{30}$

Iterative decomposition of water and fat with echo asymmetry and least squares estimation (IDEAL) ${ }^{31}$ acquires three or more echoes and uses an iterative reconstruction algorithm to decompose the chemical species, ie, fat and water, into separate images. Because IDEAL also estimates the local field map due to $\mathrm{B}_{0}$ inhomogeneities, it is more robust in the presence of $\mathrm{B}_{0}$ inhomogeneities and maximizes SNR in the reconstructed images for all combinations of the separated chemical species in a voxel.

In comparison to fat suppression with preparatory pulses, the Dixon/IDEAL techniques do not alter the image contrast and have the major advantage of being insensitive to the $\mathrm{B}_{0}$ inhomogeneities. A major drawback of the Dixon technique has been the requirement of acquiring multiple echoes and hence a longer acquisition time. However, the increased SNR from multiple echoes can be traded off with acceleration techniques such as parallel imaging and partial k-space sampling to make the acquisition faster. In case of dynamic imaging, ie, breast DCE-MRI, view sharing techniques such as time-resolved MR-angiography with stochastic trajectories (TWIST) or elliptical centric time resolved imaging of contrast kinetics (EC-TRICK) can further accelerate the acquisitions without SNR penalty. Based on this concept, view sharing accelerated fast gradient echo acquisition with dual-echo Dixon fat and water separation such as TWISTDixon $^{32}$ and differential subsampling with cartesian ordering (DISCO $)^{33}$ have been developed and have produced good results in breast DCE-MRI application.
Another alternative to avoiding longer acquisition times associated with acquiring multiple echoes of different TEs is the single-point Dixon technique. ${ }^{34}$ In single-point Dixon, the fat and water separation is based on the data from a single echo. A region growing algorithm is used for phase error correction.

Recently, several studies have been carried out to compare two-point Dixon and spectral-selective fat suppression techniques both qualitatively and quantitatively at $3 \mathrm{~T} .{ }^{35-38}$ Two-point Dixon is found to be outperforming other techniques with more uniform and complete suppression of fat signal, better anatomical definition, less artifacts, and improved contrast and visibility of the breast lesions in contrast-enhanced bilateral breast imaging. In addition to three-dimensional (3D) T1-weighted spoiled fast-gradient sequence, Dixon/IDEAL fat and water separation can also be implemented in 3D T2W fast spin echo sequence. ${ }^{39}$

\section{Slice-select gradient reversal}

Because of the chemical shift difference between fat and water, the excited fat and water signals are actually from two slices with an offset in the slice direction. If the gradient for slice-selective refocusing is reversed, ie, if the gradients for slice-selective excitation and slice-selective refocusing have opposite polarity, the refocusing of slice for fat and the refocusing of slice for water will have an offset in the opposite direction. If the total of two offsets is greater than the slice thickness, the excited fat magnetization would not be refocused and therefore has no contribution to the echo signal, ${ }^{40,41}$ as shown in Figure 12. 


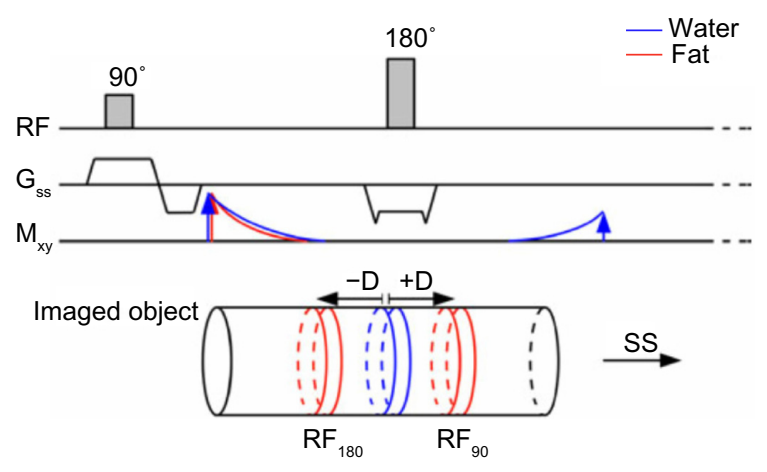

Figure 12 Schematic illustration of SSGR fat suppression.

Notes: The $90^{\circ}$ RF pulse excites fat in a slice that is slightly displaced from that of water. When the polarity of gradient is reversed, but has the magnitude for the $180^{\circ}$ refocussing pulse, the excited slice of fat is displaced in the opposite direction by the same amount. If the total shift is greater than the slice thickness, the fat signal is not refocused, while the water signal is completely refocused. Springer and European Society of Radiology. Eur Radiol, Combination of chemical suppression techniques for dual suppression of fat and silicone at diffusion-weighted MR imaging in women with breast implants. 22, 20I2, 2648-2653, Koh D, Blackledge M, Burns S, et al, CEuropean Society of Radiology 2012, with kind permission of Springer Science+Business Media. ${ }^{49}$ Abbreviations: SSGR, slice-select gradient reversal; RF, radio frequency; SS, slice selection.

Similar to other fat suppression techniques based on chemical shift, slice-select gradient reversal (SSGR) also requires uniform $\mathrm{B}_{0}$ and benefits from the greater separation of fat and water resonance frequencies at higher field strengths. Unlike CHESS and WE, which only suppress or excite signals with a certain bandwidth, SSGR actually suppresses all signals that are off-resonance, including silicone. Another advantage of SSGR is that it does not require any additional time or RF pulse. However, SSGR is limited to twodimensional (2D) SE sequence with slice-selective excitation and refocusing, such as 2D SE EPI used for breast DWI.

\section{Fat suppression at 3.0T versus I.5T}

Breast MRI at 3.0T has many advantages over 1.5T. The greater signal generated at higher field strength can be traded off through various acceleration techniques to improve the spatial and temporal resolution in breast MRI scans. Improved lesion detection may be achieved through gains in spatial resolution and greater contrast-to-noise ratio, while lesion characterization may be improved through higher spatial and/or temporal resolution. ${ }^{42}$

Despite the greater frequency separation between fat and water signal, fat suppression is in general more difficult at 3.0T than $1.5 \mathrm{~T}$ because of the increased $\mathrm{B}_{0}$ and $\mathrm{B}_{1}^{+}$inhomogeneity.

Fat suppression techniques need to be adapted to 3.0T. For spectral-selective excitation or saturation techniques such as CHESS or WE, the frequency offset and the RF pulse bandwidth need to be adjusted to the greater frequency separation between fat and water and the greater $\mathrm{B}_{0}$ inhomogeneity. Such change is usually implemented through pulse sequence design. For IR-based techniques such as STIR or SPAIR, a longer TI should be used to null the fat signal. The greatest challenge for fat suppression at higher field strength is to compensate for $\mathrm{B}_{1}{ }^{+}$inhomogeneity. ${ }^{43}$ This may be accomplished through advanced hardware such as multi-channel parallel transmission which enables $\mathrm{B}_{1}{ }^{+}$shimming. ${ }^{44}$ For IR-based techniques, switching the inversion RF pulse to an adiabatic pulse which is insensitive to $\mathrm{B}_{1}{ }^{+}$inhomogeneity can be helpful. ${ }^{23}$

\section{Fat suppression in the presence of metallic implant}

Breast MRI is often performed as follow-up exams on cancer patients to monitor for possible tumor recurrence. For patients who have had prior biopsy or surgery, biopsy markers or surgical clips are usually placed during the procedure for future references, and for patients undergoing chemotherapy, a port for vascular access may be present. These metallic implants and devices cause strong perturbation of the local $\mathrm{B}_{0}$ and present additional challenges for fat suppression by producing artifacts of complex dark and bright bands in the conventional fat-suppressed images. ${ }^{45} \mathrm{~A}$ recent study shows that metal artifact has a slightly smaller volume and more distinct pattern in Dixon water-only images than gradient echo images with QFS or SPAIR. ${ }^{46}$

\section{Fat suppression in the presence of silicone implant}

The percentage of patients with breast implants is on the rise. In 2008, more than 300,000 women and teenagers in the US underwent breast augmentation surgery and about 80,000 women underwent reconstructive breast implant surgery after mastectomy ${ }^{47}$ While MRI is recommended as the method of choice for investigating silicone gel implant rupture, ${ }^{48}$ the presence of silicone implant causes additional complexity for fat suppression in breast MRI because silicone also produces MR signal with an off-resonance frequency of $300 \mathrm{~Hz}$ at $1.5 \mathrm{~T}$, close to that of fat, and a T1 relaxation time of $900 \mathrm{~ms}$, which fortunately is much longer than fat $\mathrm{T} 1$ relaxation time. When the objective of breast MRI scan is to detect or evaluate lesions in the breast tissue, it is desirable to suppress both fat and silicone signals. In such cases, dual fat and silicone suppression can be achieved with a combination of two techniques described previously, such as STIR and SSGR or STIR and IDEAL. ${ }^{49,50}$ However, if breast MRI is performed to evaluate the integrity of the silicone implant, both fat and water signal should be suppressed while preserving the silicone signal, so any rupture or leakage of the silicone implant can be 
visualized. Then, an IR technique, such as STIR, can be used to suppress the fat signal and a spectral-selective saturation with its frequency centered at water peak can be used to suppress water signal. Another approach to achieve desired suppression of unwanted signal is to reconstruct separate water, fat, and silicone images using the Dixon method. ${ }^{51}$

\section{Optimizing fat suppression in breast MRI}

For fat suppression techniques which rely on matching the frequency of RF pulse with the frequency of fat resonance, having the $\mathrm{B}_{0}$ as uniform as possible throughout the entire breast or the anatomy of interest, and having the center frequency of the MR scanner tuned to the water frequency is critical. A practical and effective method to reduce the $\mathrm{B}_{0}$ inhomogeneity at the air and breast tissue boundary is to carefully remove the skin folds when positioning the patient. In bilateral breast MRI, the transverse images often have a larger than necessary FOV in the anterior to posterior direction. This is because the frequency encoding needs to be in the anterior to posterior direction in order to avoid ghosting artifacts of the moving heart from propagating into the breasts. By default, the shimming or optimization of $\mathrm{B}_{0}$ homogeneity is performed by the scanner over the entire large FOV instead of just the breast region. To achieve better $\mathrm{B}_{0}$ uniformity over the breasts and surrounding regions, a localized shim volume covering only the breasts, the axilla, and the chest wall can be set up manually to optimize the $\mathrm{B}_{0}$ homogeneity only for those regions and ignore other regions such as the heart and lungs, ${ }^{52}$ At the same time, the adjustment of the scanner's center frequency should be optimized for the same regions as well. Most of the MR scanners allow users to manually check or confirm the setting of center frequency after the automatic adjustment is made. It is important to do so in breast MRI. Checking center frequency manually can also prevent the occasional error of setting center frequency to fat signal when the fat signal dominates.

Another factor that can impact the quality of fat suppression is the transmitting $\mathrm{RF}$ field $\left(\mathrm{B}_{1}^{+}\right)$inhomogeneity or inaccuracy, which is worse at higher fields such as $3 \mathrm{~T}$ due to the so-called dielectric effect. On advanced MR scanners with multiple independent transmitting coils, it is possible to perform $\mathrm{B}_{1}^{+}$shimming to reduce $\mathrm{B}_{1}^{+}$inhomogeneity. However, this technique is still under development and not widely available. In the meantime, the options to reduce $\mathrm{B}_{1}^{+}$ inhomogeneity are very limited for an end user. The best thing to do is to choose a technique that is insensitive to $\mathrm{B}_{1}^{+}$ variations, such as diabetic inversion or Dixon fat and water separation.

\section{Conclusion}

Suppressing the fat signal can improve the sensitivity of breast MRI. However, accurate and time-efficient fat suppression continues to be a challenge, especially at higher field strengths where $\mathrm{B}_{0}$ and $\mathrm{B}_{1}^{+}$are less uniform. Recently, new strategies, such as SPAIR and Dixon, have been introduced and have significantly improved the quality and robustness of fat suppression in breast MRI. Combined with the latest acceleration strategies, such as parallel imaging and view sharing, the Dixon technique is capable of producing water-only images with minimal artifact and even higher SNR efficiency. Such technical advancement is alleviating the conflict between high spatial-temporal resolution and better conspicuity with fat suppression.

\section{Acknowledgment}

The authors would like to thank Hamidah Zarrinmayeh for assistance in manuscript preparation.

\section{Disclosure}

The authors have no conflicts of interest to disclose.

\section{References}

1. Centers for Disease Control and Prevention [homepage on the Internet] USA: Breast Cancer Statistics; 2014 [updated September 2, 2014]. Available from: http://www.cdc.gov/cancer/breast/statistics/. Accessed October 10, 2014

2. American Cancer Society. Cancer Facts and Figures. Atlanta: American Cancer Society; 2014. Available from: http://www.cancer.org/acs/groups/ content/@research/documents/document/acspc-042725.pdf. Accessed October 10, 2014

3. Macura KJ, Ouwerkerk R, Jacobs MA, Bluemke DA. Patterns of enhancement on breast MR images: interpretation and imaging pitfalls. Radiographics. 2006;26(6):1719-1734.

4. Mann RM, Veltman J, Barentsz JO, Wobbes T, Blickman JG, Boetes C. The value of MRI compared to mammography in the assessment of tumour extent in invasive lobular carcinoma of the breast. Eur J Surg Oncol. 2008;34(2):135-142.

5. Mann RM, Kuhl CK, Kinkel K, Boetes C. Breast MRI: guidelines from the European Society of Breast Imaging. Eur Radiol. 2008;18(7): 1307-1318.

6. Li SP, Makris A, Beresford MJ, et al. Use of dynamic contrast-enhanced $\mathrm{mr}$ imaging to predict survival in patients with primary breast cancer undergoing neoadjuvant chemotherapy. Radiology. 2011;260(1): 68-78.

7. Lee C, Dershaw DD, Kopans D, et al. Breast cancer screening with imaging: recommendations from the society of breast imaging and the acr on the use of mammography, breast mri, breast ultrasound, and other technologies for the detection of clinically occult breast cancer. J Am Coll Radiol. 2010;7(1):18-27.

8. Schnall MD, Blume J, Bluemke DA, et al. Diagnostic architectural and dynamic features at breast MR imaging: multicenter study. Radiology. 2006;238(1):42-53.

9. Kuhl C. The current status of breast MR imaging. Part I. Choice of technique, image interpretation, diagnostic accuracy, and transfer to clinical practice. Radiology. 2007;244(2):356-378. 
10. Kuhl CK, Mielcareck P, Klaschik S, et al. Dynamic breast MR imaging: are signal intensity time course data useful for differential diagnosis of enhancing lesions? Radiology. 1999;211(1):101-110.

11. Saslow D, Boetes C, Burke W, et al. American Cancer Society guidelines for breast screening with MRI as an adjunct to mammography. CA Cancer J Clin. 2007;57(2):75-89.

12. Partridge SC, McDonald ES. Diffusion weighted magnetic resonance imaging of the breast: protocol optimization, interpretation, and clinical applications. Magn Reson Imaging Clin N Am. 2013;21(3):601-624.

13. Pinker K, Bogner W, Baltzer P, et al. Improved diagnostic accuracy with multiparametric magnetic resonance imaging of the breast using dynamic contrast-enhanced magnetic resonance imaging, diffusionweighted imaging, and 3-dimensional proton magnetic resonance spectroscopic imaging. Invest Radiol. 2014;49(6):421-430.

14. Bolan PJ. Magnetic resonance spectroscopy of the breast: current status. Magn Reson Imaging Clin N Am. 2013;21(3):625-639.

15. IlcolYO1, Ozbek R, Hamurtekin E, Ulus IH. Choline status in newborns, infants, children, breast-feeding women, breast-fed infants and human breast milk. J Nutr Biochem. 2005;16(8):489-499.

16. Kerslake RW, Carleton PJ, Fox JN, et al. Dynamic gradient-echo and fat suppressed spin-echo contrast-enhanced MRI of the breast. Clin Radiol. 1995;50(7):440-454.

17. Le Y, Akisik F, Dale B, Koons K, Lin C. The Impact of Dixon Fat Suppression on Liver T1 and DCE Perfusion Quantification. Proceedings of the 21st Annual Meeting of the International Society of Magnetic Resonance in Medicine April 20-26, 2013; Salt Lake City, UT, USA.

18. Schmidt MA, Borri M, Scurr E, et al. Breast dynamic contrast-enhanced examinations with fat suppression: are contrast-agent uptake curves affected by magnetic field inhomogeneity? Eur Radiol. 2013;23(6):1537-1545.

19. Rakow-Penner R, Daniel B, Yu H, Sawyer-Glover A, Glover GH. Relaxation times of breast tissue at 1.5T and 3T measured using IDEAL. J Magn Reson Imaging. 2006;23(1):87-91.

20. Haase A, Frahm J, Hänicke W, Matthaei D. 1H NMR chemical shift selective (CHESS) imaging. Phys Med Biol. 1985;30(4):341-344.

21. Desmond KL, Ramsay EA, Plewes DB. Comparison of biphasic and reordered fat suppression for dynamic breast MRI. J Magn Reson Imaging. 2007;25(6):1293-1298.

22. Wenkel E, Geppert C, Schulz-Wendtland R, et al. Diffusion weighted imaging in breast MRI: comparison of two different pulse sequences. Acad Radiol. 2007;14(9):1077-1083.

23. Balchandani P, Spielman D. Fat suppression for $1 \mathrm{H}$ MRSI at $7 \mathrm{~T}$ using spectrally selective adiabatic inversion recovery. Magn Reson Med. 2008;59(5):980-988.

24. Morrell GR. Rapid fat suppression in MRI of the breast with short binomial pulses. J Magn Reson Imaging. 2006;24(5):1172-1176.

25. Meyer CH, Pauly JM, Macovskiand A, Nishimura DG. Simultaneous spatial and spectral selective excitation. Magn Reson Med. 1990;15(2): 287-304.

26. Zur Y. Design of improved spectral-spatial pulses for routine clinical use. Magn Reson Med. 2000;43(3):410-420.

27. Han M, Cunningham CH, Pauly JM, Daniel BL, Hargreaves BA. Homogenous fat suppression for bilateral breast imaging using independent shims. Magn Reson Med. 2014;71(4):1511-1517.

28. Dixon WT. Simple proton spectroscopic imaging. Radiology. 1984; 153(1):189-194.

29. Le-Petross H, Kundra V, Szklaruk J, Wei W, Hortobagyi GN, Ma J. Fast three-dimensional dual echo dixon technique improves fat suppression in breast MRI. J Magn Reson Imaging. 2010;31(4):889-894.

30. Mito S, Ishizaka K, Nakanishi M, Sugimori H, Hamaguchi H, Tsuzuki T. [Comparison of fat suppression techniques of bilateral breast dynamic sequence at 3.0 T: utility of three-point DIXON technique]. Nihon Hoshasen Gijutsu Gakkai Zasshi. 2011;67(6):654-660. Japanese.

31. Reeder SB, Pineda AR, Wen Z, et al. Iterative decomposition of water and fat with echo asymmetry and least-squares estimation (IDEAL): application with fast spin-echo imaging. Magn Reson Med. 2005;54(3):636-644.
32. Le Y, Kroeker R, Kipfer HD, Lin C. Development and evaluation of TWIST Dixon for dynamic contrast-enhanced (DCE) MRI with improved acquisition efficiency and fat suppression. J Magn Reson Imaging. 2012;36(2):483-491.

33. Saranathan M, Rettmann DW, Hargreaves BA, Clarke SE, Vasanawala SS. Differential subsampling with cartesian ordering (DISCO): A high spatio-temporal resolution dixon imaging sequence for multiphasic contrast enhanced abdominal imaging. J Magn Reson Imaging. 2012;35(6):1484-1492.

34. Ma J. A single-point dixon technique for fat-suppressed fast 3D gradient-echo imaging with a flexible echo time. J Magn Reson Imaging. 2008;27(4):881-890.

35. Lin C, Owens L, Kipfer H, Schultze-Haakh H, Dale B. Quantitative Evaluation of Fat Suppression Techniques for Breast MRI at 3.0T. Proceedings of the 19th Annual Meeting of the International Society of Magnetic Resonance in Medicine May 1-7, 2010; Stockholm, Sweden.

36. Dogan BE, Ma J, Hwang K, Liu P, Yang WT. T1-weighted 3D dynamic contrast-enhanced MRI of the breast using a dual-echo Dixon technique at 3 T. J Magn Reson Imaging. 2011;34(4):842-851.

37. Mikami WK, Kazama T, Sato H, et al. Fat suppression strategies in MR imaging of breast cancer at 3.0 T: comparison of the two-point Dixon technique and the frequency selective inversion method. Jpn J Radiol. 2013;31(9):615-622.

38. Clauser P, Pinker K, Helbich TH, Kapetas P, Bernathova M, Baltzer PA. Fat saturation in dynamic breast MRI at 3 Tesla: is the Dixon technique superior to spectral fat saturation? A visual grading characteristics study. Eur Radiol. 2014;24(9):2213-2219.

39. Madhuranthakam AJ, Yu H, Shimakawa A, et al. T(2)-weighted 3D fast spin echo imaging with water-fat separation in a single acquisition. J Magn Reson Imaging. 2010;32(3):745-751.

40. Tang H, Wu EX, Kennan R, Liu H, Williams DS. Interleaved water and fat imaging and applications to lipid quantitation using the gradient reversal technique. J Magn Reson Imaging. 2007;26(4): 1064-1070.

41. Nagy Z, Weiskopf N. Efficient fat suppression by slice selection gradient reversal in twice-refocused diffusion encoding. Magn Reson Med. 2008;60(5):1256-1260.

42. Rahbar H, Partridge SC, DeMartini WB, Thursten B, Lehman CD. Clinical and technical considerations for high quality breast MRI at 3 Tesla. J Magn Reson Imaging. 2013;37(4):778-790.

43. Azlan CA, Di Giovanni P, Ahearn TS, Semple SI, Gilbert FJ, Redpath TW. B1 transmission-field inhomogeneity and enhancement ratio errors in dynamic contrast-enhanced MRI (DCE-MRI) of the breast at 3T. J Magn Reson Imaging. 2010;31(1):234-239.

44. Ishizaka K, Kato F, Terae S, et al. Bilateral breast MRI by use of dualsource parallel radiofrequency excitation and image-based shimming at 3 Tesla: improvement in homogeneity on fat-suppression imaging. Radiol Phys Technol. Epub June 26, 2014.

45. Genson CC, Blane CE, Helvie MA, Waits SA, Chenevert TL. Effects on breast MRI of artifacts caused by metallic tissue marker clips. AJR Am J Roentgenol. 2007;188(2):372-376.

46. Le Y, Kipfer HD, Majidi SS, Holz S, Lin C. Comparison of the artifacts caused by metallic implants in breast MRI using dual-echo dixon versus conventional fat-suppression techniques. AJR Am J Roentgenol. 2014;203(3):W307-W314.

47. breastimplantinfo.org [homepage on the Internet]. The National Resource Center for Women and Families. What You Need to Know About Breast Implants. Available from: http://www.breastimplantinfo. org/augment/implantfacts.html. Accessed October 10, 2014.

48. Rietjens M, Villa G, Toesca A, et al. Appropriate use of magnetic resonance imaging and ultrasound to detect early silicone gel breast implant rupture in postmastectomy reconstruction. Plast Reconstr Surg. 2014;134(1):13e-20e.

49. Koh D, Blackledge M, Burns S, et al. Combination of chemical suppression techniques for dual suppression of fat and silicone at diffusionweighted MR imaging in women with breast implants. Eur Radiol. 2012;22:2648-2653. 
50. Madhuranthakam AJ, Smith MP, Yu H, et al. Water-silicone separated volumetric MR acquisition for rapid assessment of breast implants. J Magn Reson Imaging. 2012;35(5):1216-1221.

51. Geppert C, Jellus V, Kiefer B. Three images from two echoes: Reconstruction of water, fat and silicone images using a combined one-point and two-point Dixon approach. Application to 3D GRE in breast implant imaging. Proceedings of the 17th Annual Meeting of the International Society of Magnetic Resonance in Medicine 18-24 April 2009; Honolulu, Hawaii, USA.
52. Takatsu Y, Nishiyama K, Miyati T, Miyano H, Kajihara M, Akasaka T. A comparison of shimming techniques for optimizing fat suppression in MR mammography. Radiol Phys Technol. 2013;6(2):486-491.

Reports in Medical Imaging

\section{Publish your work in this journal}

Reports in Medical Imaging is an international, peer-reviewed, open access journal publishing original research, reports, reviews and commentaries on all areas of medical imaging. The manuscript management system is completely online and includes a very quick and fair peer-review system, which is all easy to use.
Dovepress

Visit http://www.dovepress.com/testimonials.php to read real quotes from published authors. 